\title{
MG II LINE PROFILES (OF THE MIRA S CARINAE
}

\author{
Edward W. Brugel \\ Center for Astrophysics \\ and Space Astronomy \\ University of Colorado \\ Boulder, Colorado
}

\author{
Jay A. Bookbinder and Alexander Brown \\ Joint Institute for Laboratory Astrophysics \\ and National Bureau of Standards \\ University of Colorado \\ Boulder, Colorado
}

\begin{abstract}
A series of high-dispersion IUE observations obtained to investigate the evolution of the shock structure of the Mira $\mathrm{S}$ Carinae (S Car) have produced - despite very limited phase coverage - a set of five exceptionally interesting spectra of the $\mathrm{Mg}$ II $\mathrm{h}$ and $\mathrm{k}$ lines. The two primary findings of these observations are 1) there is significant emission from both the $h$ and $k$ lines at velocities of $-150 \mathrm{~km} \mathrm{~s}^{-1}$ relative to the stellar photosphere and 2) the h-to-k ratio of the $\mathrm{Mg}$ II doublet remains below the theoretically predicated values of 2.1 to $1: 1$, and shows a smooth dependence on the optical phase. Archival studies of other Miras (e.g. R Car) indicate that $\mathrm{S}$ Car is not unique in possessing unusual and highly variable $\mathrm{Mg}$ II $\mathrm{h}$ and $\mathrm{k}$ line profiles.
\end{abstract}

Keywords: lines: profiles - shock waves - stars: emısszonline - stars: Miras - stars: atmospheres

\section{INTRODUCTION}

Until recently. very few attempts have been made to observe the $\mathrm{Mg}$ II resonance lines of Miras (or other evolved stars) in high dispersion. Such attempts as have been made have lacked the phase coverage that is necessary to account for the fact that Miras are long-period pulsational variables, and that the associated atmospheric changes will inevitably influence the characteristics of the Mg II line profiles $\mathrm{Un}_{\mathrm{n}}$ doubtedly, some of the difficulty of observing these stars adequately throughout the course of even one full period stems from the long timescales involved - generally on the order of one to two years. Nonetheless, vital clues regarding the formation of extended chromospheres, mass loss, and shock propagation are contained in these line profiles, especially when they are observed over a significant fraction of the pulsation cycle.

Previous IUE observations of the atmospheres of Miras and related pulsationally unstable stars (e.g., RV Tauris) have concentrated on the integrated $\mathrm{Mg}$ Il line fluxes (obtained from low-dispersion IUE observations), and in particular their variability as a function of the optıcal phase (Brugel,
Willson, and Cadmus 1986. and Brugel et al. 1987). Thcoretical models (Bowen 1988) developed to explain the phasecoupled variability and describe the mifluence of the outward propagating shock on the atmosphere, are primarily concerned with explaining the integrated $\mathrm{Mg}$ II flux observations and at present, do not attempt to describe the line profiles. Nonethcless, for at least some stars ( $e g, \mathrm{~T}$ Cephei) the agreement between the observed and predicted integrated $\mathrm{Mg}$ II line flux is quite satisfactory, though for $\mathrm{S}$ Car the agrecment is particularly poor (Brugel et al. 1987).

We have selected for a high-dispersion study this relatively short period (149 days) Mira S Carmae, which has the added attraction of an extremely high radial velocity $(+284$ $\mathrm{km} \mathrm{s}^{-1}$ ) (Willson, Wallerstein, and Pilachowski 1982) This radual velocity is sufficient to remove the $\mathrm{Mg}$ II emission entircly from the interstellar medium absorption, enabling us to obtain accurate line fluxes and profiles without the uncertainty associated with modelling the ISM absorption profile $\mathrm{S}$ Car is also bright enough in $\mathrm{Mg}$ II that high resolution LWP observations can be obtained in a reasonable amount of time throughout the bulk of the shock cycle. We present these observations, as well as some preliminary inter pretations, below.

\section{OBSERVATIONS}

A series of eight low- and six high-dispersion long wavelength $(1900-3400 \AA)$ IUE spectra were obtained of the $\mathrm{Mg}$ II $h$ and $\mathrm{k}$ lines near $2800 \AA$ during November 1987 . These observations, spaced one to 17 days apart (equivalent to 0.02 to 0.11 of the optical phase) and covering a total of three weeks ( 0.15 of the total optical cycle) are the first high-dispersion observations made with a view towards studying the phase dependence of the $\mathrm{Mg}$ II line profiles and emission strengths. Additional low and high dispersion spectra were obtained on $17 / 18$ December 1987; these data are removed by $\approx 0.3$ in phase from the November observations. At the time of the final high-dispersion spectrum, the $\mathrm{Mg}$ II flux had decreased to essentially undetectable levels. Prior to each high-dispersion spectrum, a corresponding low-dispersion observation was obtained to provide an accurate measure of the total flux in the $\mathrm{Mg}$ II 
Table II

S Carinae - UV Data

\begin{tabular}{|ccrrr|}
\hline $\begin{array}{c}\text { Optical } \\
\text { Pliase }\end{array}$ & $\begin{array}{c}\text { Total } \\
\text { Line Flux }\end{array}$ & $\begin{array}{r}\text { k Line } \\
\text { Flux }\end{array}$ & $\begin{array}{r}\text { h Line } \\
\text { Flux }\end{array}$ & $\begin{array}{r}\mathrm{k} / \mathrm{h} \\
\text { Ratio }\end{array}$ \\
\hline \hline & & & & \\
0.30 & 1.82 & 0.52 & 1.23 & 0.43 \\
0.32 & 1.91 & 0.47 & 1.10 & 0.42 \\
032 & 1.60 & 0.49 & 1.23 & 0.40 \\
034 & 1.57 & 0.41 & 1.05 & 0.39 \\
0.45 & 1.04 & $<0.31$ & 0.64 & $<0.48$ \\
0.46 & 1.00 & - & - & - \\
0.48 & 0.84 & $<0.20$ & $<0.52$ & - \\
0.60 & 0.24 & $<0.21$ & $<0.14$ & - \\
& & & & \\
\hline
\end{tabular}

R Carinae - UV Data

\begin{tabular}{|lllll|}
\hline 054 & - & 1.54 & 4.37 & 0.35 \\
0.61 & - & 0.88 & 1.39 & 0.63 \\
\hline 0.41 & - & 1.70 & 2.89 & 0.59 \\
\hline
\end{tabular}

$\dagger$ - The total $\mathrm{Mg}$ II flux is obtained from the low-dispersion images, all fluxes are in units of $10^{-12} \mathrm{ergs} \mathrm{cm}^{-2} \mathrm{~s}^{-1}$. No low-dispersion images were available for $\mathrm{R}$ Car.

\section{III.1 The Mg II Line Position}

The observed $\mathrm{Mg}$ II emission lines are not seen at the stellar rest velocity, but rather shifted to shorter wavelengths by approximately $1.6 \AA$ (assuming a stellar radial velocity of $+284 \mathrm{~km} \mathrm{~s}^{-1}$ ). There are two possible explanations for this position shift. First, the emission may be intrinsically asymmetric, with a single emission component shifted by about $150 \mathrm{~km} \mathrm{~s}^{-1}$ towards shorter wavelengths. Alternatively, the line may be symmetric about the stellar rest velocity, with width of about $300 \mathrm{~km} \mathrm{~s}^{-1}$. The second hypothesis is supported by measurements of the $\mathrm{Mg}$ II pumped $\mathrm{Fe}$ I lines at $2823.3 \AA$ and $2844.0 \AA$, which sample the $\mathrm{Mg}$ II flux at a stellar rest position of $2795.5 \AA$. The measured flux of the Fe I $2823.3 \AA$ line is $9 \times 10^{-14}$ and $5 \times 10^{-14}$ erg $\mathrm{cm}^{-2} \mathrm{~s}^{-1}$ at phases 0.32 and 0.45 . Assuming an Fe I absorption line width of $0.1 \AA$, this implies $\mathrm{Mg}$ II fluxes at $2795.5 \AA$, of $9 \times 10^{-13}$ and $5 \times 10^{-13} \mathrm{erg} \mathrm{cm}^{-2} \mathrm{~s}^{-1}$, which are significantly above the directly observed $\mathrm{Mg}$ II values, and also implies a much greater $\mathrm{Mg}$ II line width (of order 300 $\mathrm{km} \mathrm{s}^{-1}$, full width) than is directly observed (note that the variation of the $\mathrm{Fe} I$ line is in step with the variation of its fluorescing $\mathrm{Mg}$ II $\mathrm{k}$ line). In either case, it is apparent that there is a great deal more Mg II flux being produced in the post shock region than is observed (by about a factor of 4-5), implying a very substantial circumstellar absorption shell. lines. A detailed $\log$ of the IUE observations appears in Table I and a portion $(2790 \AA$ to $2810 \AA)$ of the first five high-dispersion spectra are shown in Figure 1.

The low dispersion spectra were extracted from the spatially resolved line-by-line file provided by IUESIPS and then merged. The high-dispersion data were taken directly from the standard IUESIPS merged, extracted file. The net spectra (gross - background) were then converted to a flux scale using the absolute calibration of Cassatella and Harris 1983. Though there are possible systematic errors in the absolute fluxes, the relative flux measurements are not similarly affected since all spectra were processed with the same calibration. A very weak ultraviolet continuum was detected near $2800 \AA$ for some of the low-dispersion observations; however, no UV continuum was detected at high-dispersion. It is thus unlikely that the continuum contributes to the line variability. We estimate that the uncertainty in the integrated flux measurements do not exceed $\pm 10 \%$. On the basis of the centering of the stellar image in the large aperture entrance slit during these observations, we estimate that the uncertainty in the wavelength scale is on the order of $\pm 10 \mathrm{~km} \mathrm{~s}^{-1}$.

\section{DISCUSSION}

The two major results of our high-dispersion observations concern the position of the $\mathrm{Mg}$ II emission lines in velocity space, and the $\mathrm{Mg}$ II $\mathrm{k}$ to $\mathrm{h}$ line ratio. These lines (and other emissions such as the hydrogen Balmer series) are believed to form in the post-shock region of an outwardly propagating atmospheric shock wave (for a recent review see Willson and Bowen 1985).

Table I

LWP Observing Log - S Carinae

\begin{tabular}{|cccccr|}
\hline $\begin{array}{r}\text { Date } \\
(1987)\end{array}$ & $\begin{array}{c}\text { Julian Day } \\
244+\end{array}$ & Phase & LWP & Disp & $\begin{array}{r}\text { Exp. } \\
\text { min }\end{array}$ \\
\hline \hline 4 Nov & 7103.66 & 0.30 & 12007 & $(\mathrm{LO})$ & 5 \\
& & 0.30 & 12008 & $(\mathrm{HI})$ & 130 \\
6 Nov & 7106.78 & 0.32 & 12033 & $(\mathrm{HI})$ & 195 \\
& & 0.32 & 12034 & $(\mathrm{LO})$ & 10 \\
8 Nov & 7107.65 & 0.32 & 12037 & $(\mathrm{LO})$ & 10 \\
& & 0.32 & 12038 & $(\mathrm{HI})$ & 135 \\
9 Nov & 7108.66 & 0.34 & 12043 & $(\mathrm{LO})$ & 10 \\
& & 0.34 & 12044 & $(\mathrm{HI})$ & 130 \\
26 Nov & 7125.83 & 0.45 & 12171 & $(\mathrm{LO})$ & 15 \\
& & 0.45 & 12172 & $(\mathrm{HI})$ & 127 \\
28 Nov & 7127.84 & 0.46 & 12186 & $(\mathrm{LO})$ & 15 \\
& & 0.46 & 12187 & $(\mathrm{LO})$ & 120 \\
29 Nov & 7129.97 & 0.48 & 12198 & $(\mathrm{LO})$ & 15 \\
& & 0.48 & 12197 & $(\mathrm{HI})$ & 103 \\
18 Dec & 7147.83 & 0.60 & 12303 & $(\mathrm{LO})$ & 20 \\
& & 0.60 & 12304 & $(\mathrm{HI})$ & 120 \\
\hline
\end{tabular}




\section{III.2 The $\mathrm{Mg}$ II $\mathrm{k} / \mathrm{h}$ Ratio}

Our second result is the very unusual ratio $(<0.5)$ of the $k$ $(2795.5 \AA)$ to $\mathrm{h}(2802.7 \AA)$ line fluxes that is observed. The theoretically expected ratio for the $\mathrm{k}$ to $\mathrm{h}$ line ranges from 2 (for an optically thin atmosphere) to unity (for an optically thick atmosphere) and is usually observed to be within this range for stars with "normal" chromospheres. We examined the IUE archives and discovered that the Mira R Car also has a similar line ratio. To the best of our knowledge, the only other observation of a similarly unusual $\mathrm{Mg} \mathrm{II} \mathrm{h/k}$ line ratio for a non-Mira type star is that of the N-type carbon star TX Psc. The spectrum of this star has been successfully modelled in terms of a substantial cool shell surrounding the star (Eriksson et al. 1986). It is this shell which is responsible for the absorption of the underlying $\mathrm{Mg}$ II emission. Absorption of $\mathrm{Mg}$ II emission by overlying layers is a problem that has also been discussed in detail by Bernat and Lambert (1976) for the case of $\alpha$ Ori (M2Iab). We note that interstellar MgII cannot be responsible for the weak $k$ line flux since the radial velocity of $\mathrm{S}$ Car is sufficiently large $\left(+284 \mathrm{~km} \mathrm{~s}^{-1}\right)$ to shift the interstellar absorption well away from the line (see Fig. 1).

These prcliminary observations also indicate that this ratio is varying smoothly from 0.43 to 0.39 as the optical phase progresses from 0.30 to 0.45 (see Figures 1 and 2 , and Table II). During this same period the integrated line flux of the blended $\mathrm{Mg}$ II $\mathrm{k}+\mathrm{h}$ line seen in low-dispersion decreases by nearly a factor of two. Observations of $\mathrm{R}$ Car show even more pronounced variations of the $k / h$ ratio during the course of a shock cycle, ranging between 0.63 at phase
0.61 to 0.35 at phase 0.54 . While $\mathrm{R}$ Car's $\mathrm{Mg}$ II lines are subject to absorption by the ISM, this cannot be the source of the observed variability. Even though the R Car observations were made at widely separated times, as well as the relatively poor quality of the $\mathbf{R}$ Car spectrum at phase 0.41 , we believe that the variation is real, and supports our contention that the $h / k$ ratio for $S$ Car does indeed vary during a single shock cycle.

A preliminary understanding of this variability can be had as follows. The $\mathrm{Mg}$ II $\mathrm{h}$ and $\mathrm{k}$ line absorption profiles have slightly different wings, with the $\mathrm{k}$ line absorption somewhat broader. As the shock slows down relative to the circumstellar absorbers, the $\mathrm{k}$ line will be absorbed more strongly, resulting in a decrease in the $\mathrm{k} / \mathrm{h}$ ration. This is precisely the effect that is seen, and may yield an estimate of the amount of material in the circumstellar shell.

\section{III.3 Search for Additional Shock Excited Lines}

Finally, we note that since the $\mathrm{Mg}$ II is undoubtedly formed in a post-shock cooling region, one might expect to see other shock excited semi-forbidden lines of CII] $2325 \AA$, SiII] $2335 \AA$, SiIV] $1385 \AA$, OIV] $1406 \AA$, CIII] $1909 \AA$, OIII $1663 \AA$, NIII] $1750 \AA$, and NII] $2141 \AA$. We have carefully searched for these lines in both a deep SWP and LWP exposure, and find no evidence for any of these lines except for a very marginal ( $1 \sigma)$ detection of the OIII] $1663 \AA$ line $\left(5.5 \times 10^{-15}\right.$ ergs $\left.\mathrm{cm}^{-2} \mathrm{~s}^{-1}\right)$. Lack of detection of the cooler lines, formed near the same temperatures as $\mathrm{Mg} \mathrm{II}$, is somewhat surprising, and may imply that the post-shock densities are greater than the critical densities needed to quench the emission.

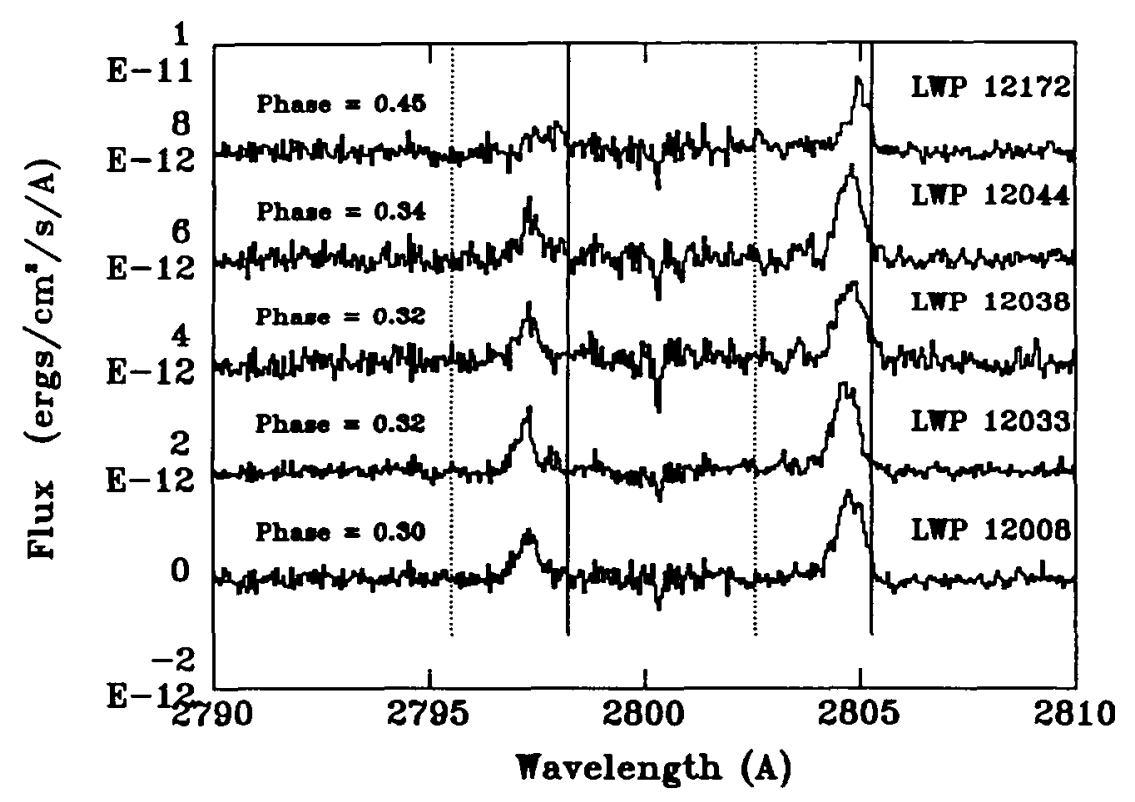

Figure 1: A series of five high dispersion spectra of $\mathrm{S}$ Car are presented. An offset in flux of $2 \times 10^{-12}$ has been added to each successive spectrum. The vertical dashed lines mark the rest wavelength of the $\mathrm{Mg}$ II lines in the laboratory frame, the vertical solid lines are in S Car's rest frame. 


\section{SUMMARY}

Several ninportant, yet prelininaty, conclusions are diawn frem the present observations. Based upon the Mg II line position and redward alsorption, there appears to be a gieat deal more $\mathrm{Mg}$ II flux produced in the post shock icgon than is observed, implying a very substantial circumstellar absorption shell In ackdition, the apparent variation of the $1 / \mathrm{k}$ line ratio during the shock cycle may be $c x-$ plained by the elative motion of the sluck to the overlying absor bing material. We note, however, that the apparent raration in the ratio of the these hnes is on the order of a $2 \sigma 1 \mathrm{csult}$, and confirmation of this result will require additional observations obtained with higher signal-to-noise clita.

The theoretical models of Bowen (1988), while they do not inclucle the racliative transfer calculations or circumstellar absorption. and hence cannot be used to obtain line profiles, do incor porate the effects of multiple shocks and dust, and have bern used to estimate line intensities for comparison with observations. However, these models must be modIfied in light of our observations that show that (1) most of the flux in the $\mathrm{Mg}$ II lines is being absorbed, implying that the cooling efficiency in the post shock region is signnficantly grcater than currently belicved, (2) the correlation of $\mathrm{Mg}$ II line flux with phase must also account for the clange in the ratio of the $\mathrm{Mg}$ II $\mathrm{h} / \mathrm{k}$ lines during the cycle; and (3) the models must now account for very redshifted cmission, as well as the lack of blue-shifted emission. It is clear that a thorough understanding of these results will find wide applicability in understanding the outer envelopes and dynamics of cool stars other than Miras.

\section{ACKNOWLEDGEMENTS}

These latia were redued and processed at the University of Colonado's IUE-RDAF, which is supported by NASA (ontı, t mumly NASJ-28731 This work was supported in pilt ly NASA contracts NAG5-82 and NAG5-350 to the Unuresity of Colorado

\section{REFERENCES}

Bernat, A.P., and Lambert. D.L, 1976, Ap.J., 204, 830.

Bohlin. R.C., and Holm. A.V., 1980, NASA IUE Newsletter. 10,37 .

Bowen, G.H . 1988 Ap.J., (n press).

Butgel, E.W., Willson, L., and Cadmus, R.. 1986, in New Insights in Astrophysucs, Proc. Joint NASA / ESA / SERC Conference, London, 14-16 July 1986, ESA SP-263, p. 213.

Biugel. E W., Beach, T., Willson, L.A., and Bowen, G., 1987, in IAU Symposium 103: The Symbrotuc Phenomena, (in press).

C'assatella. A. and Harrs, A.W., 1983, NASA IUE Newsletter, 23, 21.

Eriksson, K., et al., 1986, A.A., 161, 305

Jolnson, H. Baumert, J., Querci, F., and Querci, M., 1986, Ap $J, 311,960$.

Willson, LA and Bowen, G.H. 1985, in The Relation Between Chromospheric-coronal Heating and Mass Loss in Stars, eds. Stalio and Zirker, pg. 127-156.

Willson, L.A., Wallerstein, G., and Prlachowski, C., 1982, M.N.R.A.S., 198, 483.

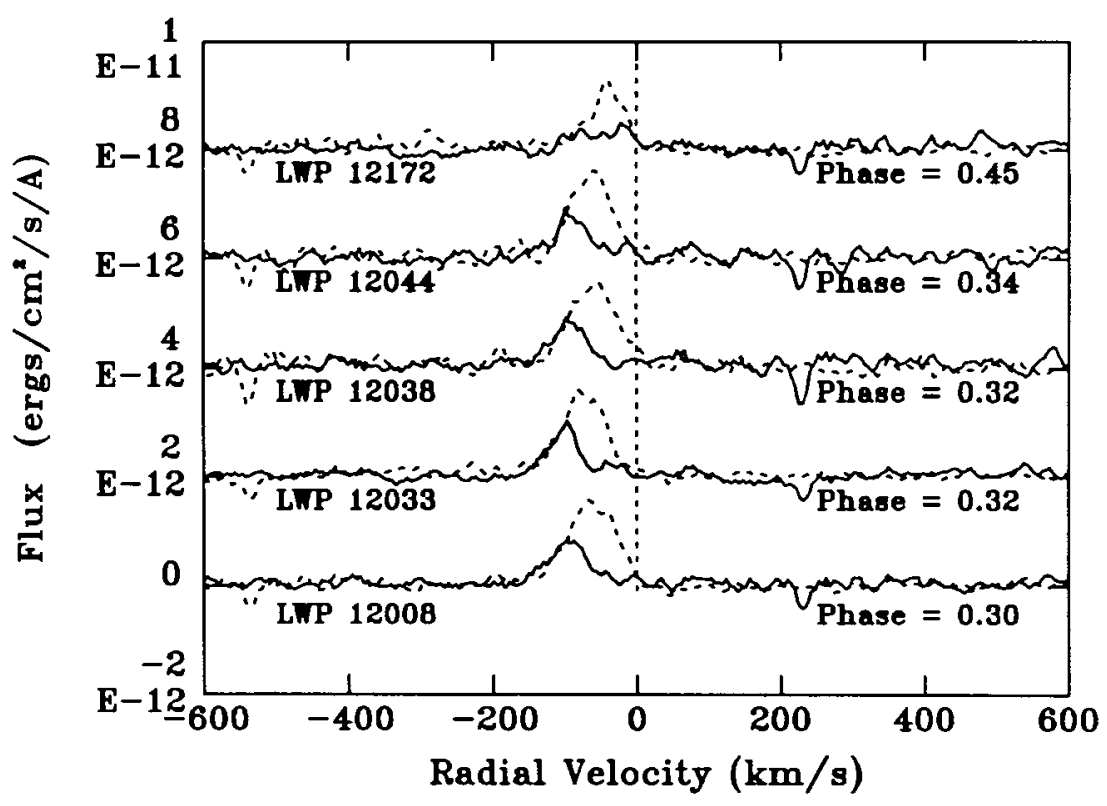

Figure 2: An overlay - in velocity space - of S Car's Mg II h (dashed) and $k$ (solid) lines for the phases in Figure 1. The spectra have been shifted by the stellar radial velocity which is $+284 \mathrm{~km} \mathrm{~s}^{-1}$. Absorption by interstellar MgII would appear close to $+284 \mathrm{~km} / \mathrm{s}$ in these plots. A reseaux is found near $+220 \mathrm{~km} / \mathrm{s}$. 